\title{
Brain function - not size - is important
}

\author{
Ozlem Taskapilioglu', M. Ozgur Taskapilioglu², Bahattin Hakyemez ${ }^{3}$, Ahmet Bekar ${ }^{2}$ \\ ${ }^{1}$ Department of Neurology, Uludag University School of Medicine, Bursa, Turkey \\ ${ }^{2}$ Department of Neurosurgery, Uludag University School of Medicine, Bursa, Turkey \\ ${ }^{3}$ Department of Radiology, Uludag University School of Medicine, Bursa, Turkey
}

\begin{abstract}
Some incidental findings on cranial imaging may render every one speechless. Brain plasticity in childhood and slow expansion of arachnoid cyst may be possible explanations of the discordance between the clinical and radiological findings. Here, we presented an asymptomatic, incidentally detected arachnoid cyst accompanied by compression of most parts of brain, displacing the motor cortex to the occipital parts of the existing brain tissue. The case was discussed in the light of the rare patients in the literature.
\end{abstract}

Eur Res J 2017;3(3):273-275

Keywords: Plasticity, arachnoid cyst, brain function

\section{Introduction}

Brain plasticity, ability of the brain to change physically, functionally, and chemically - throughout life, is one of the fully unexplained conditions of the human brain. Advanced brain plasticity in childhood may facilitate cognitive reorganization. Arachnoid cysts are benign congenital lesions that develop during the formation of the endomeninx. In this paper we presented a case of displaced motor cortex, as evident from functional magnetic resonance imaging, due to a giant asymptomatic arachnoid cyst that was detected incidentally.

\section{Case Presentation}

A 38-year-old right-handed man was admitted to the outpatient clinic with the complaint of headache that was localized to frontal region and unresponsive to the medical treatment. He was primary school graduated worker, married with two children. He had no nausea or vomiting. His neurological examination was intact. Computed tomography and magnetic resonance imaging of the brain revealed giant arachnoid cysts on the convexity of frontal lobe, occupying $17.5 \times 8 \times 9 \mathrm{~cm}$ in dimension on the right side, and $12 \times 8 \times 5.5 \mathrm{~cm}$ on the left (Figure 1). Temporal lobes were almost completely erased and volumes of the parietal lobes were reduced. Only occipital lobes were developed. Neuropsychological examination with frontal attention and memory tests revealed mild cognitive decline. His general mental capacity was limited. Speech centre showed left-sided dominance, 
shown by activation sides on functional magnetic resonance imaging (Figure 2). There is no change with his neurological status at the end of 2-year follow-up.

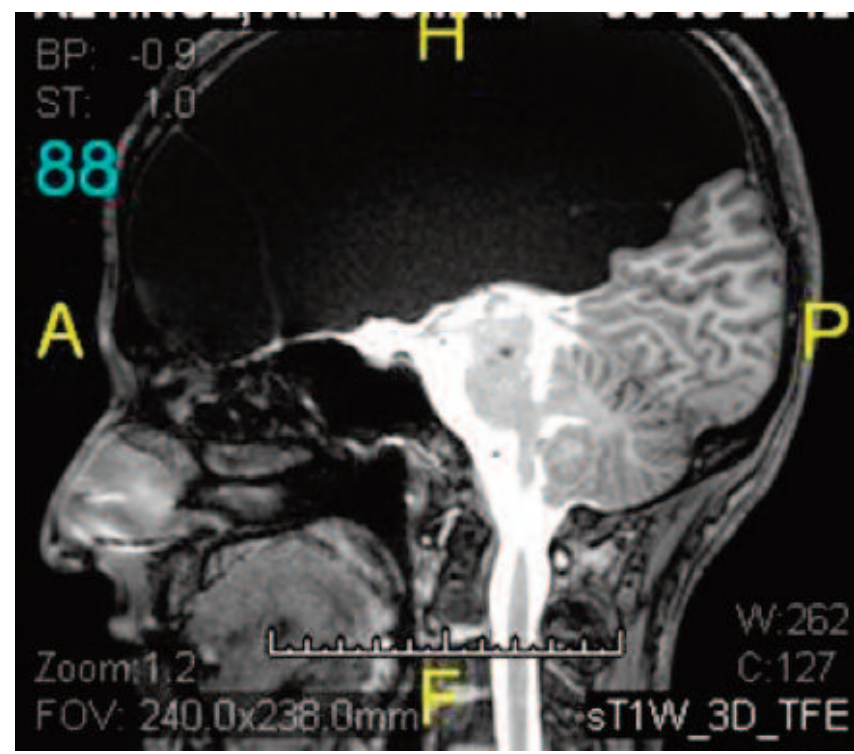

Figure 1. T2 weighted sagittal section of MRI of the brain revealed giant arachnoid cysts on the convexity of frontal lobe.

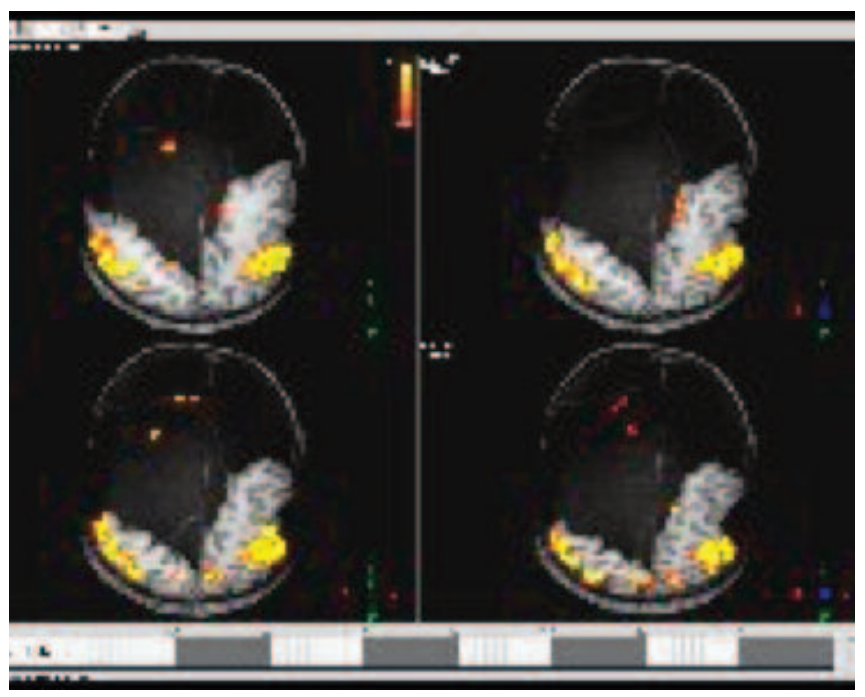

Figure 2. Speech centre showed left-sided dominance, shown by activation sides on functional MRI.

\section{Discussion}

Arachnoid cysts are cavities with content similar to cerebrospinal fluid. They are often found incidentally in $0.1-0.4 \%$ of the general population and they make up approximately $1 \%$ of all intracranial space-occupying lesions [1]. A critical point is the extent of the compression capacity of human brain by space-occupying brain lesions. Rare case illustrations, in the literature, showed that giant arachnoid cysts displaced cortical and subcortical structures which were still compatible with normal brain function [13]. Motor cortex can be displaced due to a giant asymptomatic arachnoid cyst, as in our case. We showed this with functional magnetic resonance imaging.

There was no deformation at the skull of our patient so this showed us that this arachnoid cyst hadn't created a high intracranial pressure and its growth was parallel to the developing brain. Nickel et al. [2] and Artico et al. [4] pointed that the continuum of the compression of the patients' brain in early adulthood, during which complete skull ossification had occurred. This was controversial to the findings in our patient.

The subtle neuropsychological impairment, being evident only in the attention and memory domains of the frontal lobe function, may be due to the arachnoid cyst. However, this interpretation cannot be made with absolute certainty since the patient himself did not report any difficulty in his daily life.

In a series of five patients with non-symptomatic arachnoid cysts in the left temporal fossa, the authors concluded that arachnoid cysts did not disturb the normal asymmetry of hemisphere language organization despite delicate locations adjacent to the left inferior frontal gyrus because of the absence of aphasic disturbance in their patients [5]. The topography of language activation did not differ significantly from the one in the healthy controls, although magnetic resonance morphometry of the patients' brains revealed reduction of cortical thicknesses in the left hemispheric grey matter adjacent to the cysts. In our case, the language activation sides were placed to occipital lobe.

\section{Conclusion}

The discordance between clinical and radiological findings were evident in this giant asymptomatic, incidentally detected arachnoid cyst which was accompanied by compression of most parts of brain, displacing the motor cortex to the occipital parts of the existing brain tissue. Slow expansion of this intracranial cyst may be a possible explanation of this discordance.

\section{Informed consent}

Written informed consent was obtained from the patient for the publication of this case report.

\section{Conflict of interest}


The authors declared that there are no potential conflicts of interest with respect to the research, authorship, and/or publication of this article.

\section{References}

[1] Bucuk M, Tomic Z, Bralic M, Rudez J, Jurjevic A. A case with giant arachnoid cysts manifested as migraine. Wien KlinWochenschr 2008;120:665.

[2] NickelJ, Jorgens S, Bussfeld P, Seitz RJ. Asymptomatic motor cortex displacement due to a giant arachnoid cyst. Neurocase 2008;13:328-33.

[3] Gleissner U, Sassen R, Schramm J, Elger CE, Helmstaedter C. Greater functional recovery after temporal lobe epilepsy surgery in children. Brain 2005;128:2822-9.

[4] Artico M, Cervoni L, Salvati M, Fiorenza F, Caruso R. Supratentorial arachnoid cysts: clinical and therapeutic remarks on 46 cases. Acta Neurochir (Wien) 1995;132:75-8.

[5] Hund-Georgiadis M, Yves Von Cramon D, Kruggel F, Preul C. Do quiescent arachnoid cysts alter CNS functional organization?: A fMRI and morphometric study. Neurology 2002;59:1935-9. 